(C) 2020 Elsevier Ltd. This manuscript version is made available under the CC-BY-NC-ND 4.0 license (http://creativecommons.org/licenses/by-nc-nd/4.0/)

Evaluation of supercritical fluid chromatography accurate mass spectrometry for neonicotinoid compounds determination in wine samples.

Article published in Journal of Chromatography A

DOI: https://doi.org/10.1016/j.chroma.2020.460963 


\title{
Evaluation of supercritical fluid chromatography accurate mass spectrometry for neonicotinoid compounds determination in wine samples
}

\author{
L. Pérez-Mayán, M. Cobo-Golpe, M. Ramil, R. Cela, I. Rodríguez
}

Department of Analytical Chemistry, Nutrition and Food Sciences. Institute for Research and Food Analysis (IIAA). Universidade de Santiago de Compostela, 15782-Santiago de Compostela, Spain.

\begin{abstract}
The suitability of supercritical fluid chromatography, coupled to accurate mass spectrometry (SFCMS), for the determination of seven commercial neonicotinoid pesticides and one transformation product in wine samples has been investigated. Thorough optimization of the different parameters (column, modifier, back-flush pressure, make up, etc.) affecting the SFC-MS selectivity and sensitivity was carried out. Under final conditions, a $2 \mathrm{~mL}$ volume of either red or white wine was extracted by SPE and analysed using external standard calibration achieving limits of quantification from 1 to $11 \mathrm{ng} \mathrm{mL}^{-1}$. Global recoveries ranged between 63 and 118\%, depending on the compound and the spiked amount. When compared to UPLC performance, SFC resulted in a better separation of compounds, narrower peaks, comparable sensitivity and significantly lower matrix effects. The developed method allowed the quantification of imidacloprid in around half of the 25 commercial wine samples processed, all of them proceeding from grapes cultivated in year 2018 in the Northwest of Spain, with a maximum concentration of $33 \mathrm{ng} \mathrm{mL}^{-1}$. Acetamiprid was also detected in some white wine samples.
\end{abstract}

Keywords: neonicotinoids; supercritical fluid chromatography; wine analysis; accurate mass spectrometry

${ }^{*}$ corresponding author: M. Ramil

e-mail:maria.ramil@usc.es

Tel. 0034881814466 


\section{Introduction}

Nowadays, neonicotinoids are the most employed insecticides all over the world [1-2]. They are widely used in agriculture for crops protection, but also in gardens, urban parks and even as ectoparasiticides for pets. This versatility is due to their systemic action which allows the effective distribution of active ingredients through the whole treated organism [3], and to their lower toxicity for mammals compared to insects [4]. These highly polar compounds have reached different environmental compartments such as water, soil or sediments [5-6], and although the effects of their long term exposure are still under research, an important fact has made neonicotinoids to be considered a potential threat for the environment: they have been associated to the disappearance of bees [7-8]. As a result, the open agriculture uses of three neonicotinoid congeners (clothianidin, CLO; thiamethoxam, THM; and imidacloprid, IMI) have been prohibited in EU countries since December 2018 [9]. Moreover, five out of the seven known pesticides of this family are included in the watch list of emerging pollutants to be monitored in continental waters [10].

Regarding wine production, neonicotinoids have been used to control some pests, such as leafhoppers (acetamiprid, ACE) [11] or mealybugs (IMI) [12], in vineyards, and their residues are susceptible to be transferred to wine during grapes vinification. In fact, transfer factors close to the unit have been reported for IMI and ACE [13].The EU rules the maximum residue levels (MRLs) of authorized neonicotinoids in vinification grapes [14]; however, as happens for other pesticides concentrations in wine are not regulated. In a previous study, we have reported the presence of IMI residues in white wines elaborated in the Northwest of Spain before year 2016 [15]. Another study has found similar low concentrations of this compound in commercial wines, whilst those of imidacloprid-olefin (IMI-OLE), considered as a major metabolite of IMI, reached up to 20-times higher levels that those of the precursor insecticide [16].

Several neonicotinoid insecticides, particularly those now banned in the EU, have been included in different multiclass pesticide LC-MS (MS/MS) determination methods based on a generic sample treatment method such as QuEChERS [17-18], reversed-phase SPE [19-20], or even direct injection after dilution and filtration of the wine sample [21]. Usually, these methods are affected by 
strong and compound-dependent signal suppression effects; thus, compounds quantification requires the use of matrix-matched standards. Rodriguez-Cabo et al. [15] have carried out the selective extraction of five neonicotinoids in red and white wines using two different SPE sorbents. During extraction, compounds were retained using an OASIS HLB cartridge; thereafter, in the elution step, a cartridge of Florisil was on-line connected with the first sorbent. Acetonitrile was passed through both sorbents releasing target compounds, whilst more polar interferences and red wine pigments are trapped by the Florisil cartridge. Such approach provided quantitative extraction yields, with very low variations in the efficiency of ESI ionization between wine extracts and solvent-based standards.

Supercritical fluid chromatography (SFC), based on packed columns, has been reported to improve selectivity, sample throughput and to reduce solvent consumption compared to LC [22]. When coupled to MS, the ESI desolvation process is more favorable due to the presence of $\mathrm{CO}_{2}$ gas, instead of an aqueous environment; moreover, the composition and the flow of the mobile phase and the make-up can be tuned to enhance sensitivity and to avoid matrix effects [23-24]. On the other hand, in the most popular SFC-MS interface, the flow of mobile phase is diluted with the make-up solution and divided into two streams before entering the ESI source. This feature might increase the LOQs of SFC-MS methods versus LC-MS [25]. Concerning pesticides analysis, SFCMS parameters have been recently optimized for the determination of hundreds of residues with different polarities providing higher sensitivity and selectivity than LC-MS [26] and also reducing matrix effects [27]. In the case of neonicotinoids, however, just very few SFC-MS applications, focused on a unique compound [28-29], have been reported. In summary, the applicability of SFCESI-MS vs LC-ESI-MS for the sensitive determination of the currently known neonicotinoids in the complex wine matrix has not been evaluated systematically, yet.

The aim of this work is to characterize the performance of SFC-ESI-MS for the determination of the so far commercialized neonicotinoids (seven species), and the potential metabolite of IMI (IMIOLE), in wine samples. SFC conditions are systematically optimized and the performance of the resulting SFC-ESI-MS method is compared to that obtained by reversed-phase UPLC-ESI-MS, 
based on the same MS platform consisting of an ESI source and a quadrupole time-of-flight (QTOF) mass analyser. Thereafter, sample preparation conditions, based on SPE, are reevaluated for the set of selected compounds. Finally, the analytical features of the whole analytical procedure (SPE followed by SFC-ESI-MS) are characterized and the presence of neonicotinoid residues in commercial wines, elaborated in the campaign of 2018 , is discussed.

\section{Material and methods}

\subsection{Chemicals and solvents}

Standards of dinotefuran (DIN), ACE, CLO, IMI, IMI-OLE, nitenpyram (NITE), thiacloprid (THC), and THM were acquired from Sigma-Aldrich (Milwaukee, WI, USA). The internal surrogates (ISs) IMI- $d_{4}$ and CLO- $d_{3}$ were also purchased from Sigma-Aldrich. THM- $d_{4}$ was acquired from Biozol (Eching, Germany). Chemical structures of native and labelled compounds, together with their Log $\mathrm{K}_{\mathrm{ow}}$ values, are provided as supplementary material, Fig. S1. Individual solutions of each compound (1000 $\mathrm{g} \mathrm{m} \mathrm{m}^{-1}$ ) were prepared in methanol $(\mathrm{MeOH})$. Concentrated solutions were protected from light in amber vessels and stored at $-20^{\circ} \mathrm{C}$. Further dilutions and calibration mixtures of the above compounds were made in acetonitrile. An internal surrogate mixture with the three isotopically labelled analytes, at $1 \mu \mathrm{g} \mathrm{mL}^{-1}$, was used for spiking wine samples, extracts and solvent-based calibration standards. The concentration of ISs was maintained at $50 \mathrm{ng} \mathrm{mL}^{-1}$.

$\mathrm{MeOH}$ and acetonitrile, both with HPLC-grade purity, glacial formic acid (FA) (98\%), ammonia and acetic acid were supplied by Merck (Darmstadt, Germany). Ultra-pure deionized water (18.2 M $\Omega$ $\mathrm{cm}^{-1}$ ) was obtained from a Milli-Q Gradient A-10 system (Millipore, Billerica, MA, USA). OASIS HLB and Florisil cartridges (containing 200 and 900 mg of sorbent, respectively) were provided by Waters (Milford, MA, USA). 


\subsection{Samples and sample preparation}

Wine samples, both red and white, were obtained from different supermarkets and also from ongoing research projects related to pesticide analysis in wines elaborated from ecological production grapes. These samples were stored at room temperature and protected from direct light exposure till extraction.

Different SPE conditions were tested for the extraction, and the potential clean-up, of neonicotinoids from wine samples. During these studies, breakthrough problems were investigated by passing the spiked samples through two OASIS HLB cartridges connected in series. After the concentration step, cartridges were eluted separately. The volume of solvent required to recover the compounds from SPE cartridges was evaluated by collecting $2 \mathrm{~mL}$ fractions of the elution solvent. Florisil was tested as potential clean-up sorbent to improve the selectivity of compounds extraction from the wine matrix.

Under optimized conditions, wine samples $(2 \mathrm{~mL})$ were diluted with the same volume of ultrapure water and passed through the OASIS HLB sorbent, previously conditioned with acetonitrile and ultrapure water $(2 \mathrm{~mL}$, each). Thereafter, the cartridge was rinsed with $2 \mathrm{~mL}$ of ultrapure water and dried with a gentle stream of nitrogen. Finally, compounds were recovered with $2 \mathrm{~mL}$ of acetonitrile. This extract was injected directly in the chromatographic system (SFC or UPLC) without any further treatment or dilution, except filtration (0.22 $\mu \mathrm{m}$ pore size).

\subsection{SFC/UPLC-MS determination conditions}

A supercritical fluid chromatographic system (SFC), model Agilent 1260 infinity II (Wilmington, DE, USA), and an ultra-performance liquid chromatographic system (UPLC), model Agilent 1290 Infinity II were employed as separation techniques. Both systems were coupled to an Agilent QTOF instrument (model 6550 Agilent), furnished with an ion funnel dual spray ESI source. The UPLC column was directly connected to the ESI source using a peek tube. The mobile phase emerging from the SFC column was mixed with the make-up solution and then split in two streams. 
One of them is connected to the back-flush pressure regulator (BPR), responsible to maintain $\mathrm{CO}_{2}$ under supercritical conditions, and the other to the ESI source. Usually, this interface is referred as pre-BPR splitter [22]. The connection between the splitting point and the ESI source was made using a $500 \mathrm{~mm}$ (length) $\times 0.05 \mathrm{~mm}$ (i.d.) silica tube provided by Agilent.

Unless otherwise stated, the QTOF instrument was operated in the MS mode. Presence of target compounds in non-spiked wines was confirmed with a second injection, using a target MS/MS method to record the product ion scan spectra of tentative identifications derived from MS spectra and retention time match with standards. The accurate MS (or product ion scan) spectra were registered in the range of $\mathrm{m} / \mathrm{z}$ values from 50 to 1700 , throughout the entire chromatogram. The TOF-MS analyser was employed in the $2 \mathrm{GHz}$ mode, achieving a spectral resolution of 15000 (data calculated at FWHM for ion at $\mathrm{m} / \mathrm{z}$ 118). Chromatograms (SFC and UPLC) were recorded at a frequency of $4 \mathrm{~Hz}$, with each spectrum corresponding to 1990 scans. The mass window employed to obtained the extracted ion chromatogram (EIC) of each compound was of $20 \mathrm{ppm}$ centred in their $[\mathrm{M}+\mathrm{H}]^{+}$ions, Table 1.

Three different columns were tested for SFC separations. They were a Viridis, ethylene bridged silica (BEH), a Torus diol, and a Torus 2-PIC (2-picolylamine). All of them were purchased from Waters. Column dimensions and particle size were: $100 \mathrm{~mm} \times 3 \mathrm{~mm}, 1.7 \mu \mathrm{m}$, in all cases. UPLC separations were carried out using a reversed-phase Zorbax Eclipse XDB $\mathrm{C}_{18}$ column (50 mm x $2.1 \mathrm{~mm}, 1.8 \mu \mathrm{m}$ ), acquired from Agilent Technologies. During analysis of wine extracts, the SFC and the reversed-phase UPLC columns, were connected to a pre-column (2.1 $\mathrm{mm}$ i.d.) containing the same phase as the analytical one.

Compounds were ionized operating the ESI source in positive mode. Nitrogen (99.999\%) was employed as nebulization (35 PSI) and drying gas $\left(15 \mathrm{~L} \mathrm{~min}^{-1}, 250^{\circ} \mathrm{C}\right)$ in the ionization source. The sheath gas $\left(\mathrm{N}_{2}\right)$ was set at $350^{\circ} \mathrm{C}$ and $11 \mathrm{~L} \mathrm{~min}-1$. The needle and fragmentor voltages were 3500 $\mathrm{V}$ and $380 \mathrm{~V}$, respectively. Above parameters were common to SFC and reversed-phase LC methods. The reference ions for recalibration of the $\mathrm{m} / \mathrm{z}$ axis in the TOF-MS system were 121.0509 and 922.0098. 
Under optimized conditions, the mobile phase used in the SFC consisted of $\mathrm{CO}_{2}$ (phase A), and $\mathrm{MeOH}$ (organic modifier), the latter containing a $5 \mathrm{mM}$ concentration of ammonium acetate as additive (phase B). The mobile phase flow rate was $1.5 \mathrm{~mL} \mathrm{~min} \mathrm{~m}^{-1}$. The chromatographic gradient was the following: 0-1 min, 2\% B; 4-6 min, 30\% B, 7-10 min, 2\% B. The make-up solution consisted of ultrapure (Milli-Q) water: $\mathrm{MeOH}(75: 25)$ at $0.2 \mathrm{~mL} \mathrm{~min}^{-1}$. The volume of injection was varied between 0.5 and $5 \mu \mathrm{L}$ depending on the experiment. Under final working conditions, $3 \mu \mathrm{L}$ was adopted as injection volume. The temperature of the SFC column was $45^{\circ} \mathrm{C}$ and the BPR was maintained at 140 bar and $60^{\circ} \mathrm{C}$.

The mobile phase used in the UPLC column consisted of water (phase A) and acetonitrile (phase B), both $0.1 \%$ in FA. The column temperature was set at $40^{\circ} \mathrm{C}$ and the flow rate of mobile phase was $0.4 \mathrm{~mL} \mathrm{~min}-1$. The percentage of acetonitrile in the mobile phase was increased from $2 \%$ to $100 \%$ in $7 \mathrm{~min}$; thereafter, the column was re-equilibrated with a $2 \%$ of acetonitrile for 3 min until the next injection.

\subsection{SPE recoveries, matrix effects and overall extraction efficiencies}

The recoveries of the extraction step were calculated as the ratio between responses obtained for wine aliquots fortified (200 $\mathrm{ng} \mathrm{mL}^{-1}$ ) before the extraction step, and for the SPE extracts (from nonspiked fractions of the same matrices) fortified at the equivalent concentration level. Obtained ratios were multiplied by $100 \%$.

Potential matrix effects (MEs,\%) during $\mathrm{ESI}(+)$ ionization were defined as the ratios between the slopes of calibration curves obtained with spiked SPE extracts from red and white wines, and those corresponding to solvent-based standards in the range of concentrations from 10 to $200 \mathrm{ng} \mathrm{mL}^{-1}$. The obtained ratios were multiplied by 100 . Values below and above $100 \%$ point out a reduction or an enhancement in the efficiency of $\mathrm{ESI}(+)$ ionization for sample extracts versus solvent-based standards. On the other hand, values around $100 \%$ suggest similar ionization efficiencies in both cases. 
Overall extraction efficiencies (accounting for the yield of the SPE step and MEs) were calculated using a solvent-based standard calibration curve built in the range LOQ-500 $\mathrm{ng} \mathrm{mL}^{-1}$, with the ISs at a level of $50 \mathrm{ng} \mathrm{mL}^{-1}$. Signals of all analytes were corrected with that for IMI- $\mathrm{d}_{4}$, excepting CLO and THM which were corrected with signals for CLO- $d_{3}$ and THM- $\mathrm{d}_{4}$, respectively.

Concentrations existing in wine extracts were calculated against solvent-based standards, after correcting the response for each analyte with that obtained for the corresponding IS. The identity of the residues found in wine samples was confirmed by comparison of the product ion scan spectra of suspected insecticides with those corresponding to authentic standards. Positive identifications require retention times and mass errors lower than $0.1 \mathrm{~min}$ and $20 \mathrm{ppm}$, respectively. In addition, mass errors of two intense product ions (see Table 1) must fit the above requirement, related to mass error, for confirmatory purposes. Given that sensitivity of TOF mass analyzers is affected by the number of scans accumulated in each spectrum [30], and considering also that SFC produces relatively narrow peaks; product ion scan spectra of suspected peaks were recorded in a second injection of wine extracts.

\section{Results and discussion}

\subsection{Optimization of the SFC conditions}

\subsubsection{Selection of type of column, column temperature and $\mathrm{CO}_{2}$ modifier}

The first step during optimization of the SFC methodology was testing the efficiency of different columns for the separation of neonicotinoid compounds. A chromatographic run with duration of 10 min, using $\mathrm{MeOH}$ as $\mathrm{CO}_{2}$ modifier, and $0.1 \% \mathrm{FA}$ as additive, was employed. The mobile phase flow was $1.5 \mathrm{~mL} \mathrm{~min}{ }^{-1}$, the injection volume $0.5 \mu \mathrm{L}$, and the column temperature $45^{\circ} \mathrm{C}$. Figure 1 shows the chromatograms obtained with the three different polar columns. As observed, they present different selectivity for the studied neonicotinoids. Both Torus columns are designed to provide great flexibility in the determination of acidic and basic compounds; however, the structural similarity of neonicotinoids made difficult their chromatographic resolution using these phases. Thus, using the Torus-2-PIC column, three compounds: DIN, THM and NITE are not completely 
resolved (Fig. 1A); using the Torus diol column there are also three peaks: IMI, NITE, THC overlapped (Fig. 1B). The silica column was the one providing the best separation of neonicotinoids (Fig. 1C), and therefore it was selected for the rest of the study. Next parameter to be optimized was the column temperature. Three different values were considered: 25,35 and 45 ${ }^{\circ} \mathrm{C}$. Despite of $\mathrm{CO}_{2}$ being under subcritical conditions at $25^{\circ} \mathrm{C}$, this temperature was evaluated based on a previous work of Kather et al [31], who obtained an appropriate separation of five pharmaceutical compounds with symmetric peaks at this temperature. However, in our case, peaks, especially those for ACE and NITE, were asymmetrical with big tails, and compounds were not properly separated using subcritical conditions (figure not shown). Best performance was achieved at $45^{\circ} \mathrm{C}$, and this was the selected temperature for the rest of the study.

After that, further experiments were carried out considering alternative modifiers and additives in the mobile phase. In comparison to $\mathrm{MeOH}$, acetonitrile led to longer retention times and poor separation of target compounds (figure not shown). The use of ammonium acetate, $5 \mathrm{mM}$, instead of FA (0.1\%) as additive did not affect the chromatographic behaviour of any compound except NITE. In this case, a slight reduction in the retention time of the compound was observed, eluting between THM and IMI-OLE; however, the highest effect was an improvement in the peak shape as well as higher repeatability in the retention time of NITE. Thus, the baseline peak width of NITE passed from 8 to $5 \mathrm{~s}$ when changing the mobile phase additive from FA to ammonium acetate. For the rest of compounds, baseline peak width values ranged between 3 and $4 \mathrm{~s}$ for both additives, considering an injection volume of $0.5 \mu \mathrm{L}$. Another advantage of replacing FA by ammonium acetate was a significant enhancement in the responses obtained for the $[\mathrm{M}+\mathrm{H}]^{+}$ion of most compounds, Fig. 2.

The chromatographic separation of neonicotinoids using the reversed-phase UPLC column, under conditions described in section 2.3, is shown in the supplementary section (Fig. S2A.). Retention times of target compounds ranged from $1.4 \mathrm{~min}$ for DIN to $2.48 \mathrm{~min}$ for ACE, with a good chromatographic separation except in case of THM and IMI-OLE, which co-eluted. The plot of 
retention times in UPLC versus SFC (BEH column) showed lack of correlation, which demonstrates the orthogonal character of both techniques, Fig. S2B.

\subsubsection{Back-flush pressure and injection volume}

Another parameter to optimize was the pressure set in the BPR of the SFC system. The higher the pressure the higher $\mathrm{CO}_{2}$ polarity, which originated a slight decrease in the retention times of selected compounds, although without affecting either peak resolution, or the selectivity of the separation. On the other hand, an increase in the back-flush pressure increases the flow rate of mobile phase reaching the ESI source in detriment of that directed to the restrictor. As a result, the obtained responses (peak areas) of all compounds increased with the back-flush pressure, Fig. S3. A value of 140 bar was selected for the rest of the study.

The next studied SFC parameter was the injection volume. Different injection volumes in the range 0.5-5 $\mu \mathrm{L}$ were tested. Both peak height and peak area linearly increase with the injection volume. On the other side, the increase in the peak width with the injected volume was only slight in the range from 0.5 to $3 \mu \mathrm{L}$. For the lower volume, the average value of the baseline peak width stayed around $3.5 \mathrm{~s}$. Considering $3 \mu \mathrm{L}$, this value increased to $4.5 \mathrm{~s}$.

\subsubsection{Make-up optimization.}

The make-up composition and flow might affect the efficiency of the ionization process. Fig.3 compares the responses obtained for different make-up solutions at a flow of $0.2 \mathrm{~mL} \mathrm{~min}^{-1}$. Ammonium acetate in $\mathrm{MeOH}$ led to a slight reduction in the observed responses in comparison to pure $\mathrm{MeOH}$; however, a noticeable improvement in the observed responses was obtained when using mixtures of $\mathrm{MeOH}: \mathrm{H}_{2} \mathrm{O}$. On the basis of these data, taking into account the consumption of organic solvent, the content of $\mathrm{MeOH}$ in the make-up solution was limited to $25 \%$. Water is less volatile than $\mathrm{MeOH}$; therefore, the desolvation efficiency is not so favoured during the ionization process and a reduction in the observed responses was expected. So, the enhanced responses in presence of water are likely due to a more efficient ionization of neonicotinoid compounds. 
Re-evaluation of the make-up flow rate, for the above solution, confirmed a reduction in the observed responses with the make-up flow rate (Fig. S4). A value of $0.2 \mathrm{~mL} \mathrm{~min}^{-1}$ was maintained since it provided a balance between stable ionization conditions and sensitivity.

\subsection{Performance of SFC-ESI-MS}

The linearity in the response of the SFC-ESI-MS system was evaluated with standards in acetonitrile, at 7 different concentration levels, in the range from 1 to $500 \mathrm{ng} \mathrm{mL}^{-1}$. The plot of responses (peak areas for the $[\mathrm{M}+\mathrm{H}]^{+}$ion of each compound) versus concentration followed a linear model, with determination coefficients $\left(R^{2}\right)$ higher than 0.996 , Table 1 . The instrumental LOQs varied from 1 to $10 \mathrm{ng} \mathrm{mL}^{-1}$. The higher LOQs corresponded to IMI-OLE, the compound rendering the wider peak in the SFC chromatograms, and DIN. Chromatographic traces for the $[\mathrm{M}+\mathrm{H}]^{+}$ion of each compound at their instrumental LOQs are provided as supplementary information, Fig. S5.

Considering the same calibration range, UPLC-ESI-MS provided similar $\mathrm{R}^{2}$ values (data not shown) to those obtained using SFC. Comparison of UPLC-MS and SFC-MS separation efficiency was performed with the baseline peak width values for neonicotinoid compounds. For standard solutions in acetonitrile, considering an injection volume of $3 \mu \mathrm{L}$, higher average baseline peak widths were observed under reversed-phase UPLC conditions than using SFC (6.5 versus $4.5 \mathrm{~s}$, respectively). Comparison of normalized responses obtained by SFC-MS and UPLC-MS, for replicate $(n=5)$ injections of the same standard are given as supplementary information (Fig. S6). Using peak areas, SFC provided higher responses (1.3 to 2.8 times) than reversed-phase UPLC for all compounds, except NITE. Working with peak height values, SFC/UPLC response ratios were even higher (from 1.5 for IMI-OLE to 5.2 for CLO) due to the fact that SFC rendered higher separation efficiency (narrower peaks) than UPLC. So, despite dilution of the SFC column stream with the make-up solution, a more efficient ionization was obtained in SFC-MS than for UPLC-MS. 


\subsection{Optimization of the wine extraction protocol}

The starting point for the optimization of the SPE conditions was a previous protocol for the determination of five of the eight compounds considered in this study in wine samples [15]. In brief, $10 \mathrm{~mL}$ of wine, diluted with the same volume of ultrapure water, were concentrated using a HLB cartridge. Thereafter, the sorbent was washed with $5 \mathrm{~mL}$ of $\mathrm{a} \mathrm{H}_{2} \mathrm{O}$ : ethanol (88:12) solution and dried with a stream of nitrogen. The HLB cartridge was on-line connected to a Florisil one. Compounds were eluted using $5 \mathrm{~mL}$ of acetonitrile passed through the dual cartridge system.

Using these initial conditions, very low extraction recoveries (below 10\%) were achieved for the three additional compounds (DIN, NITE and IMI-OLE) included in this research. Breakthrough problems were noticed for DIN and NITE when concentrating $10 \mathrm{~mL}$ of wine. Moreover, IMI-OLE was only partially recovered from the clean-up Florisil cartridge with acetonitrile $(5 \mathrm{~mL})$. In view of the above problems, the wine volume was reduced to $2 \mathrm{~mL}$, the HLB cartridge was rinsed with ultrapure water $(5 \mathrm{~mL}$ ) instead of the previously described hydro-alcoholic solution (some of the analytes were lost when using ethanol: $\mathrm{H}_{2} \mathrm{O}$ (12:88) in the rinsing step), and the on-line clean-up step was not considered. Under these conditions, the volume of acetonitrile in the elution step was reduced to $2 \mathrm{~mL}$. In summary, SPE served just as extraction technique, removing some components of the wine matrix before injection, but without providing any concentration of the samples.

The recoveries of the above procedure were evaluated in red and white wines spiked at a relatively high concentration level (200 $\mathrm{ng} \mathrm{mL}^{-1}$ ). The obtained values varied from $87 \%$ to $113 \%$ with standard deviations in the range of 1 to $11 \%$, Table 2. Matrix effects were estimated with spiked SPE extracts of red and white wines obtained from vines managed under ecological production. Slopes of addition curves for spiked extracts (addition level from 10 to $200 \mathrm{ng} \mathrm{mL}^{-1}$ ) were divided by those obtained for acetonitrile-based standards prepared in the same range of concentrations. Using SFC-ESI-MS, MEs (response ratios multiplied by 100) varied between $73 \%$ to $105 \%$, so little signal attenuation was noticed for wine extracts in comparison to solvent-based standards, Table 2. In case of UPLC-ESI-MS, the obtained MEs stayed between 35 and 92\%, depending on the 
compound and the type of wine. The earlier eluting compounds in the reversed-phase column (DIN, NITE and THM) present an average signal attenuation of 50\%. The response of CLO was also significantly attenuated (around $40 \%$ signal suppression) in wine extracts. In summary, MEs data in Table 2 show that SFC-ESI-TOF-MS was less affected by signal suppression effects than UPLC-ESI-TOF-MS

\subsection{Performance of the overall method}

The global recoveries of the process (SPE followed by SFC-ESI-MS), accounting for SPE extraction efficiencies and MEs in the ESI source, were calculated with red and white wines fortified at three concentration levels: 10,20 and $50 \mathrm{ng} \mathrm{mL}^{-1}$. Spiked and non-spiked aliquots of each wine were processed in triplicate, with the ISs added to samples at a concentration of $50 \mathrm{ng}$ $\mathrm{mL}^{-1}$. Recoveries calculated against solvent-based standards, after IS correction, are shown in Table 3. Obtained values ranged between 74 and 118\%, just NITE shows slightly lower recoveries at the lowest concentration level (63-67\%). Globally, data in Table 3 indicate that solvent-based standards are a suitable calibration option to calculate the concentrations of these compounds in wine samples.

The LOQs of the analytical procedure (SPE extraction followed by SFC-ESI-MS) are in the range from 1 to $11 \mathrm{ng} \mathrm{mL}^{-1}$, referred to the wine matrix, Table 3. These LOQs are comparable to those (1 $\mathrm{ng} \mathrm{mL}^{-1}$ ) obtained by direct large volume injection liquid chromatography tandem mass spectrometry (LC-MS/MS) [16] and slightly higher than those $\left(0.2 \mathrm{ng} \mathrm{mL}^{-1}\right)$ reported by our group using a pre-concentration step prior to LC-MS/MS [15]. These two latter methods have been developed using QqQ instruments, which are supposed to be more sensitive than TOF-MS systems.

\subsection{Determination of neonicotinoid residues in wine}

Once the method was validated, it was applied to the determination of the target neonicotinoids in a set of 25 commercial wines (8 red wines and 17 white ones) produced in the Northwest of Spain from grapes cultivated in year 2018. Around half of the investigated wines (all of them white wines 
from the same geographic denomination) contained concentrations of IMI above its LOQ. The maximum residue of this insecticide was $33 \mathrm{ng} \mathrm{mL}^{-1}$, a level which represent only $3 \%$ of the maximum permitted concentration for IMI in vinification grapes produced until 2018, Table 4. Regarding the rest of compounds, ACE was the only species noted, although at concentrations below its LOQ, in samples codes 8 and 9. IMI values in Table 4 are similar to those previously obtained for wines produced in the same area in previous campaigns $[15,19]$. IMI-OLE, whose presence has been associated to IMI residues in wine samples [16], remained below the LOQs of the developed method in all samples.

Fig. 4 shows the peak of IMI in a positive sample (Fig. 4A), together with the accurate MS (containing also the ions of the IS IMI- $\left.\mathrm{d}_{4}\right)($ Fig. 4B) and product ion scan spectra (Fig. 4C) of the compound. Differences between experimental and library $\mathrm{m} / \mathrm{z}$ values of the most abundant product ions $(\mathrm{m} / \mathrm{z} 209.0589$ and 175.0978$)$ in the product ion scan spectrum stayed below $2 \mathrm{ppm}$.

\section{Conclusions}

In this study, a method based on the use of SFC as separation technique coupled to accurate mass spectrometry has been developed for the determination of seven neonicotinoid insecticides and a transformation product in wine samples. The combination of this technique with a previous SPE extraction step provided LOQs between 1 and $11 \mathrm{ng} \mathrm{mL} \mathrm{m}^{-1}$, referred to wine samples. SFC produced narrower peaks and higher retention than reversed-phase UPLC for the set of investigated compounds. The organic extract obtained from the SPE cartridge is directly compatible with the mobile phase employed in the SFC column, and equal or lower signal attenuation effects (depending on the considered compound) were observed in comparison to UPLC-MS determination. So, despite a more complex optimization of instrumental parameters, SFC-MS is a more convenient technique than reversed-phase UPLC for the determination of the set of currently known neonicotinoid insecticides in wine samples. Further monitoring studies are required to investigate the impact of the current restriction of neonicotinoids in the presence of IMI in wine, and the potential use of alternative neonicotinoids as ACE, in wines elaborated from grapes produced after 2018. 


\section{Acknowledgements}

This study was supported by Xunta de Galicia and Spanish Government through grants GRCED431C 2017/36 and PGC2018-094613-B-I00, both co-funded by the EU (FEDER). L. P-M acknowledges a FPU contract to the Spanish Ministry of Education (FPU16/03942). 


\section{Captions to figures}

Figure 1. SFC-MS chromatograms for the $[\mathrm{M}+\mathrm{H}]^{+}$ion of each neonicotinoid on the following columns; A)Torus-2-PIC, B) Torus diol and C) Viridis BEH. (1: ACE; 2: DIN; 3: THM; 4: NITE; 5: IMI; 6: THC; 7: CLO; 8: IMI-OLE).

Figure 2. Effect of the mobile phase additive in the normalized responses of neonicotinoid compounds in SFC-MS chromatograms, $n=3$ replicates.

Figure 3. Responses obtained as function of the composition of the make-up solution $\left(0.2 \mathrm{~mL} \mathrm{~min}^{-}\right.$ $\left.{ }^{1}\right)$. Peak areas normalized to those obtained for $\mathrm{MeOH}: \mathrm{H}_{2} \mathrm{O}(25: 75), \mathrm{n}=3$ replicates.

Figure 4. Extracted ion (mass window 20 ppm) SFC-MS chromatogram (A), MS spectrum (B), and product ion scan spectra of a peak assigned to IMI in a non-spiked wine sample (code 3, Table 4). 


\section{References}

[1] P. Jeschke, R. Nauen, M. Schindler, A. Elbert, Overview of the status and global strategy for neonicotinoids, J Agric. Food Chem. 59 (2011) 2897-2908. https://doi.org/10.1021/jf101303g.

[2] M. R. Douglas, J. F. Tooker, Large-scale deployment of seed treatments has driven rapid increase in use of neonicotinoid insecticides and preemptive pest management in U. S. field crops, Environ. Sci. Technol. 49 (2015) 5088-5097. https://doi.org/10.1021/es506141

[3] N. Simon-Delso, V. Amaral-Rogers, L. P. Belzunces, J. M. Bonmartin, M. Chagnon, C. Downs, L. Furlan, D. W. Gibbons, C. Giorio, V. Girolami, D. Goulson, D. P. Kreutzweiser, C. H. Drupke, M. Liess, E. Long, M. McField, P. Mineau, E. A. D. Mitchell, C. A. Morrissey, D. A. Noome, L. Pisa, J. Settele, J. D. Stark, A. Tapparo, H. Van Dyck, J. Van Praagh, J. P. Van der Sluijs, P. R. Whitehorn, M. Wiemers, Systemic insecticides (neonicotinoids and fipronil): trends, uses, mode of action and metabolites, Environ. Sci. Pollut. Res. 22 (2015) 5-34. https://doi.org/10.1007/s11356-014-3470-y

[4] D. Goulson, An overview of the environmental risks posed by neonicotinoid insecticides, J. Appl. Ecol. 50 (2013) 977-987. https://doi.org/10.1111/1365-2664.12111.

[5] J. Bonmatin, D. A. Noome, H. Moreno, E. A. D. Mitchell, G. Glauser, O. S. Soumana, M. B. van Lexmond, F. Sánchez-Bayo, A survey and risk assessment of neonicotinoids in water, soil and $\begin{array}{lllll}\text { sediments of } \quad \text { Belize, } & \text { Environ. } & \text { Pollut. } & 249 & \text { (2019) }\end{array}$ https://doi.org/10.1016/j.envpol.2019.03.099

[6] H. A. Craddock, D. Huang, P. C. Turner, L. Quirós-Alcalá, D. C. Payne-Sturges, Trends in neonicotinoid pesticide residues in food and water in the United States, 1999-2015. Environ. Health 18 (2019) 7. https://doi.org/10.1186/s12940-018-0441-7

[7] N. Tsvetkov, O. Samson-Robert, K. Sood, H. S. Patel, D. A. Malena, P. H. Gajiwala, P. Maciukiewicz, V. Fournier, A. Zayed, Chronic exposure to neonicotinoid reduces honey bee health near corn crops, Science 356 (2017) 1395-1397. https://doi.org/10.1126/science.aam7470. 
[8] B. A. Woodcock, J. M. Bullock, R. F. Shore, M. S. Heard, M. G. Pereira, J. Redhead, L. Ridding, H. Dean, D. Sleep, P. Henrys, J. Peyton, S. Hulmes, L. Hulmes, M. Sárospataki, C. Saure, M. Edwards, E. Genersch, S. Knäbe, R. F. Pywell, Country-specific effects of neonicotinoid pesticides on honey bees and wild bees, Science $356 \quad$ (2017)1393-1395. https://doi.org/10.1126/science.aaa1190

[9] Commision Implementing Regulation (EU) No 783/2018, Off. J. Eur. Union. L132 (2018) 13

[10] Commission Implementing Decision (EU) 2018/840, Off. J. Eur. Union L141 (2018) 9

[11] N. Prabhaker, S. Castle, F. Byrne, T. J. Henneberry, N. C. Toscano, Establishment of baseline susceptibility data to various insecticides for glassy-winged sharpshooter, Homalodisca coagulate (Homoptera: Cicadellidae) by comparative bioassay techniques, J. Econ. Entomol. 99 (2006) 141154. https://doi.org/10.1093/jee/99.1.141

[12] N. Prabhaker, C. Gispert, S. J. Castle, Baseline susceptibility of Planococcus ficus (Hemiptera: Pseudococcidae) from California to selected insecticides, J. Econ. Entomol 105 (2012) 1392-1400. https://doi.org/10.1603/EC11340

[13] T. Pazzirota, L. Martín, M. Mezcua, C. Ferrer, A. R. Fernández-Alba, Processing factor for a selected group of pesticides in a wine-making process: distribution of pesticides during grape processing, Food Addit. Contam. Part A $30 \quad$ (2013) 1752-1760. https://doi.org/10.1080/19440049.2013.815806

[14] https://ec.europa.eu/food/plant/pesticides/max residue levels en. Accessed December 2019.

[15] T. Rodriguez-Cabo, J. Casado, I. Rodriguez, M. Ramil, R. Cela, Selective extraction and determination of neonicotinoid insecticides in wine by liquid chromatography-tandem mass spectrometry, J. Chromatogr. A 1460 (2016) 9-15. https://doi.org/10.1016/j.chroma.2016.07.004

[16] J. D. Berset, S. Mermer, A. E. Robel, V. M. Walton, M. L. Chien, J. A. Field, Direct residue analysis of systemic insecticides and some of their relevant metabolites in wines by liquid 
$\begin{array}{llllllll}\text { chromatography-mass } & \text { spectrometry, } & \text { J. } & \text { Chromatogr. } & \text { A } & 1506 & \text { (2017) } & \text { 45-54. }\end{array}$ https://doi.org/10.1016/j.chroma.2017.05.019

[17] J. He, B. Zhang, H. Zhang, L. Hao, T. Ma, J. Wang, S. Han, Monitoring of 49 pesticides and 17 mycotoxins in wine by QuEChERS and UHPLC-MS/MS analysis, J. Food Sci. 84 (2019) 26882697. https://doi.org/10.1111/1750-3841.14695

[18] J. Wang, W. Cheung, UHPLC/ESI-MS/MS determination of 187 pesticides in wine, J. AOAC Int. 99 (2016) 539-557. https://doi.org/10.5740/jaoacint.15-0265

[19] G. Castro, L. Pérez-Mayán, T. Rodríguez-Cabo, I. Rodríguez, M. Ramil, R. Cela, Multianalyte, high-throughput liquid chromatography tandem mass spectrometry method for the sensitive determination of fungicides and insecticides in wine, Anal. Bioanal. Chem. 410 (2018) 1139-1150. https://doi.org/10.1007/s00216-017-0724-9

[20] P. Perez-Ortega, B. Gilbert-Lopez, J. F. Garcia-Reyes, N. Ramos-Martos, A. Molina-Diaz, Generic sample treatment method for simultaneous determination of multiclass pesticides and mycotoxins in wines by liquid chromatography-mass spectrometry, J. Chromatogr. A 1249 (2012) 32-40. https://doi.org/10.1016/j.chroma.2012.06.020

[21] J. V. Dias, M. P. Nunes, I. R. Pizzutti, B. Reichert, A. A. Jung, C. D. Cardoso, Simultaneous determination of pesticides and mycotoxins in wine by direct injection and liquid chromatographytandem mass spectrometry analysis, Food Chem. $293 \quad$ (2019) 83-91. https://doi.org/10.1016/j.foodchem.2019.04.088

[22] Colin F. Poole, Supercritical Fluid Chromatography. Handbooks in separation sicence. Elsevier, 2017. Chapter 8. Pages 229-238.

[23] C. West, E. Lesellier, Effects of mobile phase composition on retention and selectivity in achiral supercritical fluid chromatography, J. Chromatogr. A 1302 (2013) 152-162. https://doi.org/10.1016/j.chroma.2013.06.003 
[24] C. West, J. Melin, H. Ansouri, M. M. Metogo, Unravelling the effects of mobile phase additives in supercritical fluid chromatography. Part I: Polarity and acidity of the mobile phase, J. Chromatogr. A 1492 (2017) 136-143. https://doi.org/10.1016/j.chroma.2017.02.066

[25] V. Pilarová, K. Plachká, M. A. Khalikova, F. Svec, L. Nováková, Recent developments in supercritical fluid chromatography-mass spectrometry: Is it a viable option for analysis of complex samples? Trac-Trends Anal. Chem. 112 (2019) 212-225. https://doi.org/10.1016/j.trac.2018.12.023

[26] Y. Fujito, Y. Hayakawa, Y. Izumi, T. Bamba, Importance of optimizing chromatographic conditions and mass spectrometric parameters for supercritical fluid chromatography/mass $\begin{array}{llllll}\text { spectrometry, } & \text { J. } & \text { Chromatogr. } & \text { A } & 1508 & \text { (2017) }\end{array}$ https://doi.org/10.1016/j.chroma.2017.05.071

[27] S. Wang, P. Qi, S. Di, J. Wang, S. Wu, X. Wang, Z. Wang, Q. Wang, X. Wang, C. Zhao, Q. Li, Significant role of supercritical fluid chromatography-mass spectrometry in improving the matrix effects and analytical efficiency during multi-pesticides residue analysis of complex chrysanthemum samples, Anal. Chim. Acta $1074 \quad$ (2019) 108-116. https://doi.org/10.1016/j.aca.2019.04.063

[28] Z. Chen, F. Dong, S. Li, Z. Zheng, Y. Xu, J. Xu, X. Liu, Y. Zheng, Response surface methodology for the enantioseparation of dinotefuran and its chiral metabolite in bee products and environmental samples by supercritical fluid chromatography/tandem mass spectrometry, J. Chromatogr. A 1410 (2015) 181-189. https://doi.org/10.1016/j.chroma.2015.07.067

[29] R. Li, Z. Chen, F. Dong, J. Xu, X. Liu, X. Wu, X. Pan, Y. Tao, Y. Zheng, Supercritical fluid chromatographic-tandem mass spectrometry method for monitoring dissipation of thiacloprid in greenhouse vegetables and soil under different application modes, J. Chromatogr. B 1081-1082 (2018) 25-32. https://doi.org/10.1016/j.jchromb.2018.02.021

[30] A.R. Fontana, I. Rodríguez, M. Ramil, J. C. Altamirano, R. Cela, Solid-phase extraction followed by liquid chromatography quadrupole time-of-flight tandem mass spectrometry for the 
selective determination of fungicides in wine, J. Chromatogr. A 1218 (2011) 2165-2175. https://doi.org/10.1016/j.chroma.2011.02.025

[31] S. Khater, C. West, E. Lesellier. Characterization of five chemistries and three particle sizes of stationary phases used in supercritical fluid chromatography, J. Chromatogr. A 1319 (2013) 148159. https://doi.org/10.1016/j.chroma.2013.10.037 

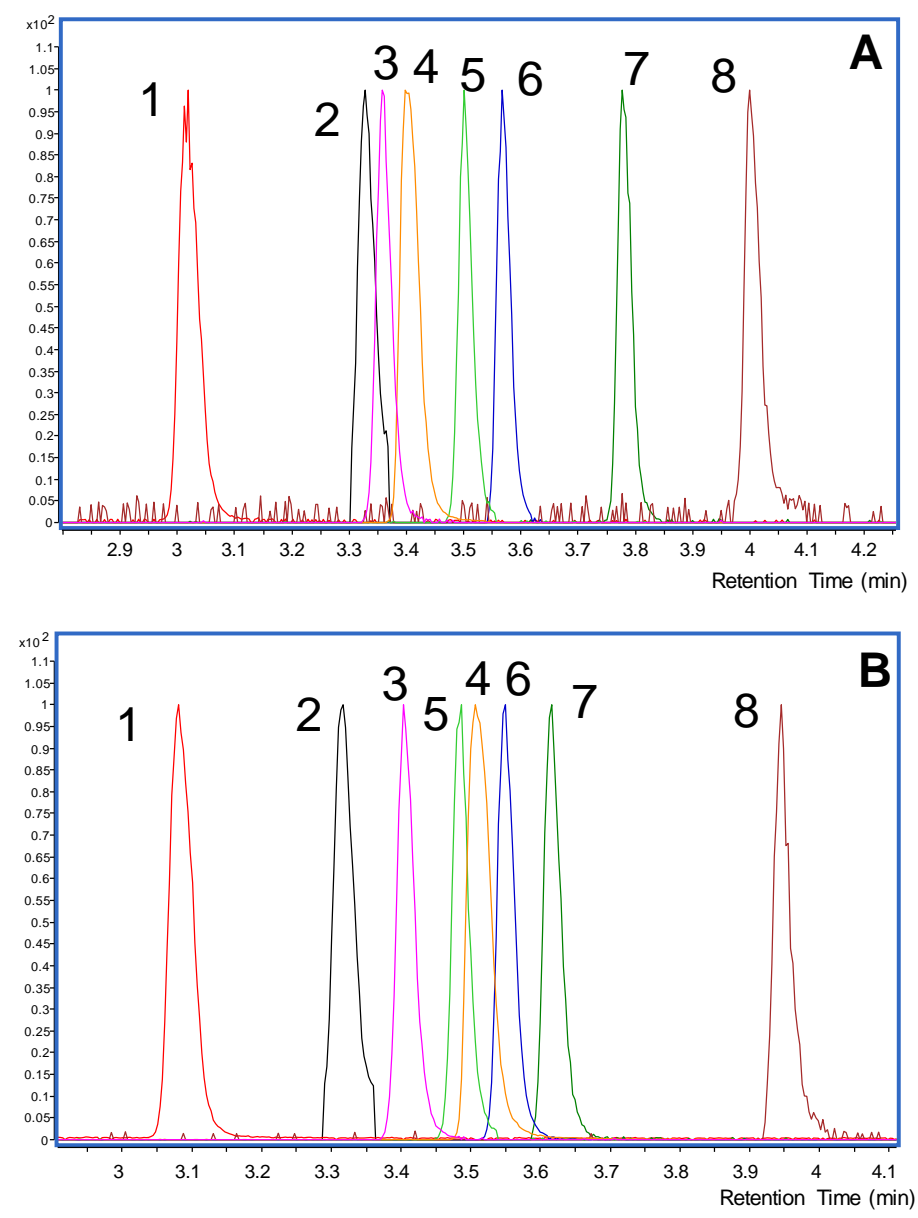

Fig. 1

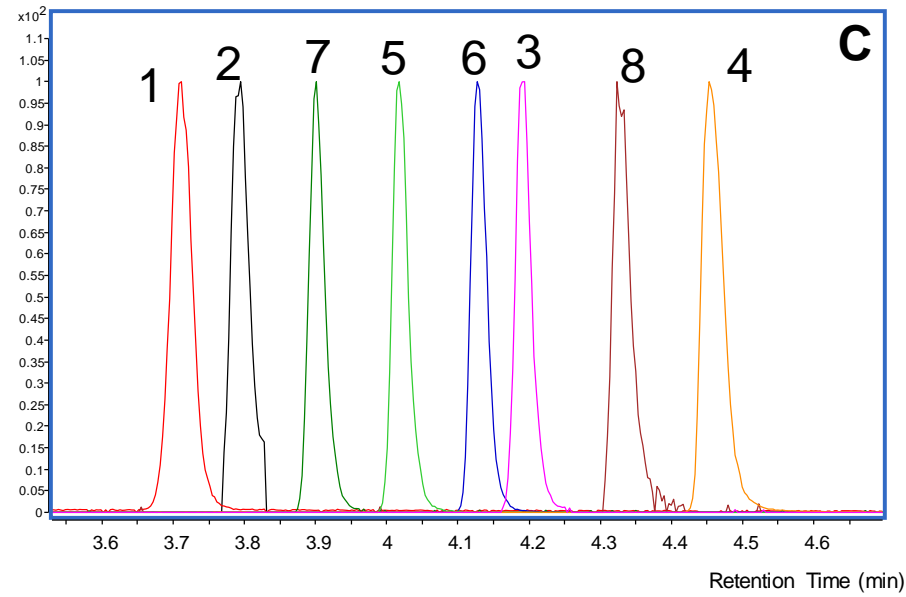




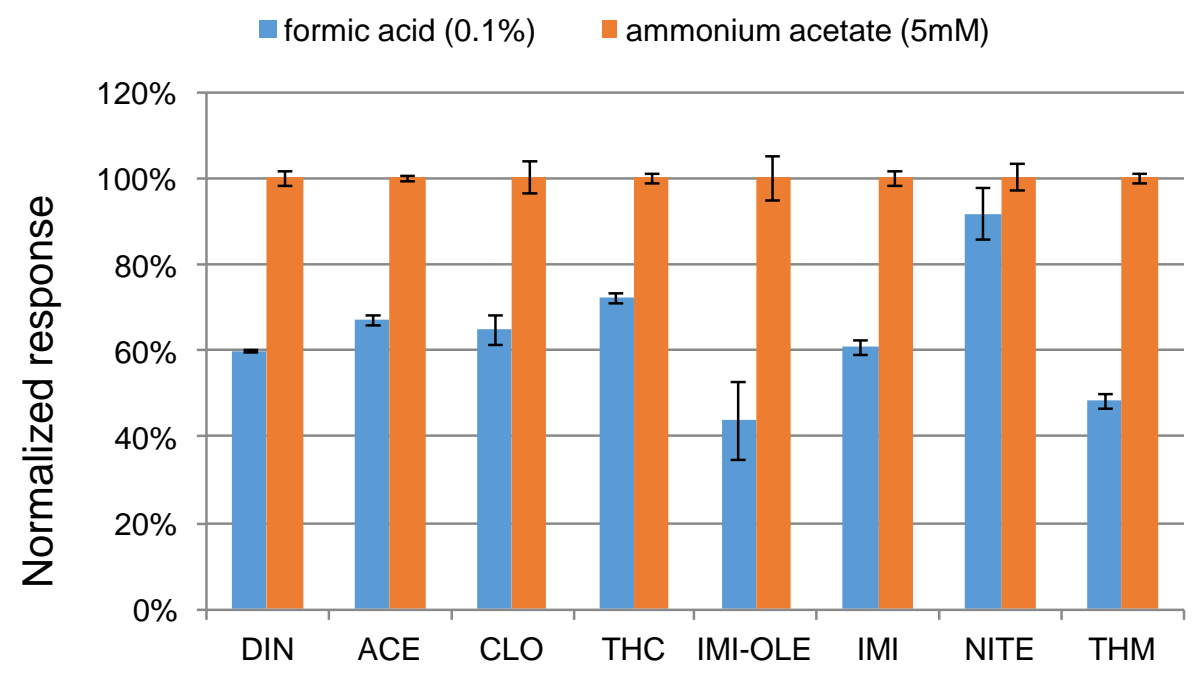

Fig. 2 


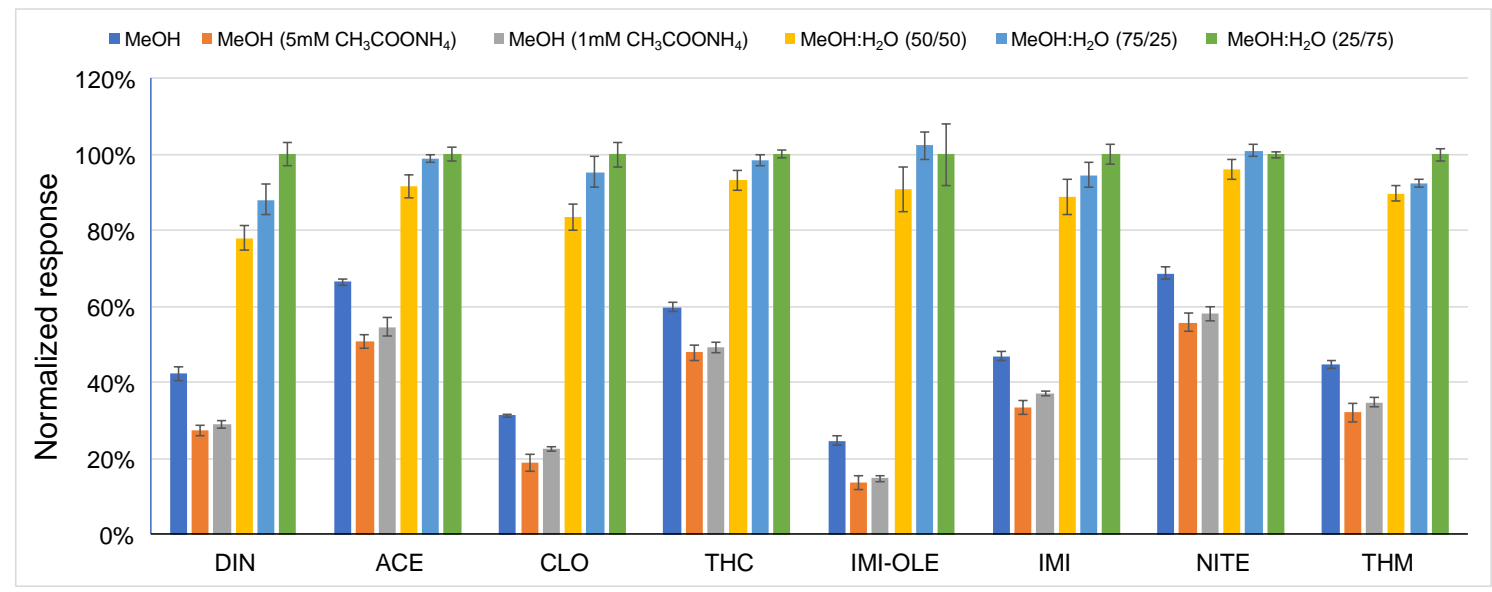

Fig. 3 

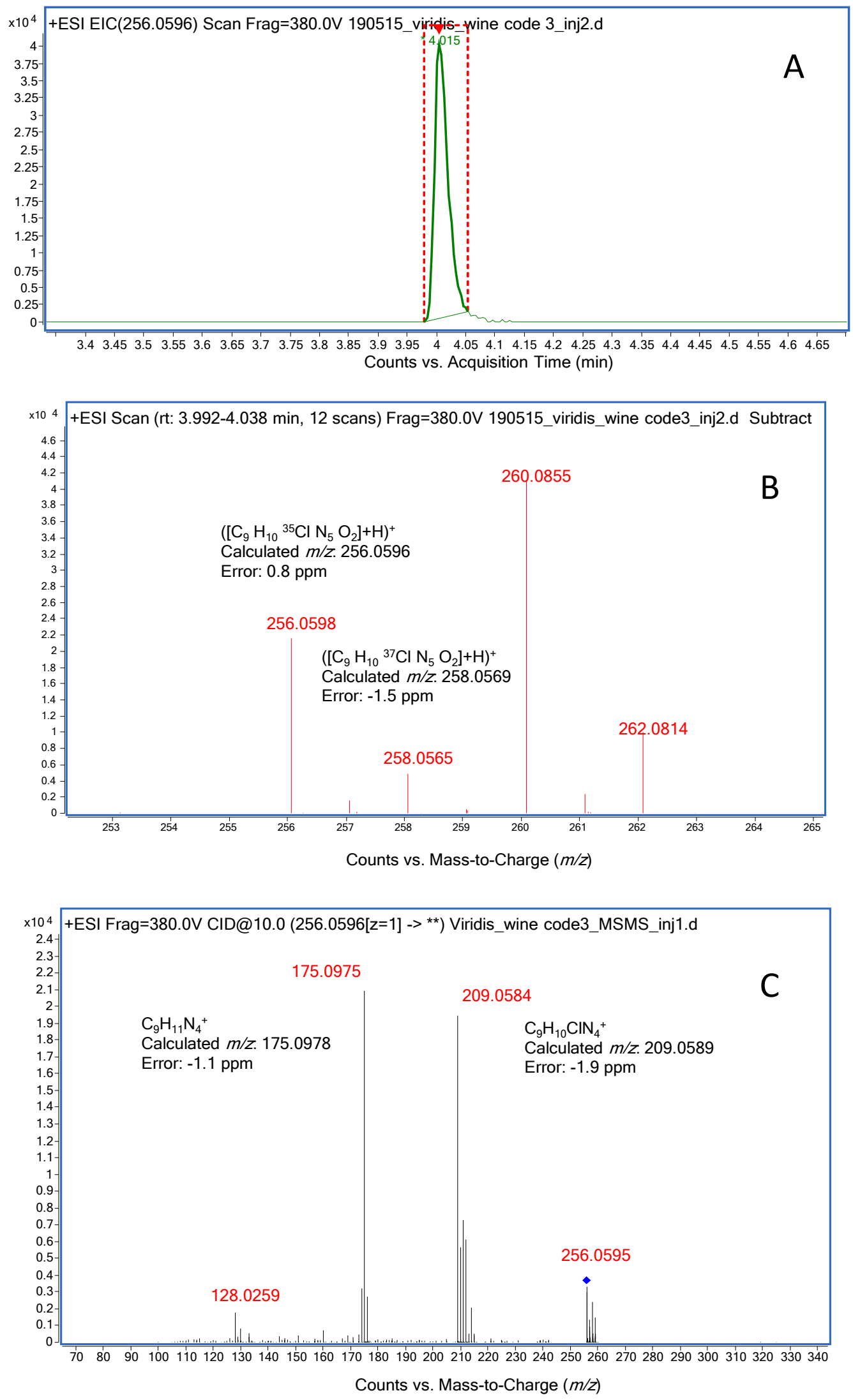

Fig. 4 
Table 1. Names, quantification ions, SFC retention times, determination coefficients $\left(\mathrm{R}^{2}\right)$, instrumental LOQs of the SFC-ESI-MS system for standards in acetonitrile, and MS/MS data for confirmatory purposes.

\begin{tabular}{|c|c|c|c|c|c|c|c|}
\hline Compound & Abbreviation & {$[\mathrm{M}+\mathrm{H}]^{+}$} & $\begin{array}{l}\text { Retention } \\
\text { Time (min) }\end{array}$ & $\begin{array}{c}\text { aLinearity } \\
\left(R^{2}\right)\end{array}$ & $\begin{array}{c}\text { LOQ } \\
\text { (ng mL }{ }^{-1} \text { ) }\end{array}$ & $\begin{array}{c}\text { Collision } \\
\text { energy } \\
(\mathrm{eV})\end{array}$ & $\begin{array}{l}\text { Most intense } \\
\text { product ions }\end{array}$ \\
\hline Dinotefuran & DIN & 203.1139 & 3.793 & 0.9986 & 10 & 10 & $129.0901,87.0783$ \\
\hline Acetamiprid & ACE & 223.0745 & 3.715 & 0.9978 & 5 & 20 & $126.0103,56.0494$ \\
\hline Clothianidin & CLO & 250.0160 & 3.909 & 0.9960 & 1 & 10 & $169.0539,131.9666$ \\
\hline Thiacloprid & THC & 253.0309 & 4.132 & 0.9978 & 1 & 15 & $186.0134,126.0106$ \\
\hline Imidacloprid-olefin & IMI-OLE & 254.0439 & 4.431 & 0.9962 & 10 & 10 & $236.0328,171.0663$ \\
\hline Imidacloprid & IMI & 256.0596 & 4.016 & 0.9989 & 1 & 10 & $209.0585,175.0979$ \\
\hline Nitenpyram & NITE & 271.0956 & 4.356 & 0.9986 & 1 & 15 & $225.1025,99.0917$ \\
\hline Thiamethoxam & THM & 292.0266 & 4.160 & 0.9981 & 1 & 20 & $211.0634,181.0545$ \\
\hline Clothianidim- $\mathrm{d}_{3}$ & $\mathrm{CLO}-\mathrm{d}_{3}$ & 253.0348 & 3.909 & & & & \\
\hline Imidacloprid- $\mathrm{d}_{4}$ & IMI-d 4 & 260.0847 & 4.023 & & & & \\
\hline Thiamethoxam- $\mathrm{d}_{4}$ & $\mathrm{THM}^{-\mathrm{d}_{4}}$ & 296.0517 & 4.160 & & & & \\
\hline
\end{tabular}

a Linearity range: LOQ-500 $\mathrm{ng} \mathrm{mL}^{-1}$ 
Table 2. SPE recoveries (\%, $\mathrm{n}=3$ replicates) under optimized conditions and comparison of matrix effects (MEs) obtained by SFC and UPLC.

\begin{tabular}{ccccccc}
\hline \multirow{2}{*}{ Compound } & \multicolumn{2}{c}{ SPE recoveries (\%) } & \multicolumn{2}{c}{ SFC MEs (\%) } & \multicolumn{2}{c}{ UPLC MEs (\%) } \\
& White wine & Red wine & White wine & Red wine & White wine & Red wine \\
\hline DIN & $104 \pm 15$ & $101 \pm 4$ & $91 \pm 3$ & $86 \pm 2$ & $40 \pm 1$ & $52 \pm 1$ \\
ACE & $88 \pm 1$ & $104 \pm 7$ & $90 \pm 3$ & $81 \pm 1$ & $75 \pm 1$ & $76 \pm 1$ \\
CLO & $91 \pm 4$ & $104 \pm 5$ & $79 \pm 3$ & $73 \pm 2$ & $51 \pm 4$ & $66 \pm 2$ \\
THC & $89 \pm 4$ & $109 \pm 1$ & $79 \pm 6$ & $74 \pm 3$ & $89 \pm 2$ & $87 \pm 1$ \\
IMI-OLE & $94 \pm 4$ & $100 \pm 11$ & $88 \pm 3$ & $102 \pm 3$ & $73 \pm 4$ & $92 \pm 3$ \\
IMI & $92 \pm 4$ & $113 \pm 10$ & $88 \pm 3$ & $76 \pm 3$ & $67 \pm 3$ & $72 \pm 1$ \\
NITE & $87 \pm 2$ & $96 \pm 1$ & $105 \pm 5$ & $88 \pm 2$ & $35 \pm 4$ & $61 \pm 1$ \\
THM & $98 \pm 9$ & $110 \pm 5$ & $92 \pm 4$ & $87 \pm 2$ & $45 \pm 2$ & $49 \pm 4$ \\
\hline
\end{tabular}


Table 3. Overall recoveries (\%), with standard deviations, for wine samples spiked at different concentration levels ( $n=3$ replicates) and LOQs of the procedure.

\begin{tabular}{cccccccc}
\hline \multirow{2}{*}{ Compound } & \multicolumn{2}{c}{ a50 $\mathrm{ng} \mathrm{mL}^{-1}$} & \multicolumn{2}{c}{${ }^{\mathrm{a}} 20 \mathrm{ng} \mathrm{mL}^{-1}$} & \multicolumn{2}{c}{ a10 $\mathrm{ng} \mathrm{mL}^{-1}$} & LOQ \\
& White & Red & White & Red & White & Red & (ng mL-1) \\
& wine & Wine & wine & Wine & wine & Wine & $\left(\mathrm{ng}^{-1}\right.$ \\
\hline DIN & $85 \pm 4$ & $75 \pm 3$ & $109 \pm 12$ & $93 \pm 9$ & - & - & 11 \\
ACE & $97 \pm 2$ & $77 \pm 2$ & $114 \pm 9$ & $94 \pm 5$ & $80 \pm 7$ & $77 \pm 4$ & 6 \\
CLO & $85 \pm 5$ & $98 \pm 3$ & $93 \pm 13$ & $74 \pm 10$ & $77 \pm 9$ & $76 \pm 8$ & 1.2 \\
THC & $103 \pm 3$ & $90 \pm 2$ & $81 \pm 8$ & $93 \pm 4$ & $85 \pm 4$ & $79 \pm 2$ & 1.1 \\
IMI-OLE & $93 \pm 6$ & $81 \pm 11$ & $103 \pm 12$ & $84 \pm 8$ & - & - & 11 \\
IMI & $118 \pm 2$ & $92 \pm 1$ & $104 \pm 5$ & $101 \pm 5$ & $110 \pm 2$ & $92 \pm 1$ & 1.0 \\
NITE & $88 \pm 1$ & $83 \pm 3$ & $102 \pm 7$ & $111 \pm 7$ & $67 \pm 5$ & $63 \pm 4$ & 1.1 \\
THM & $93 \pm 4$ & $92 \pm 3$ & $91 \pm 10$ & $94 \pm 5$ & $93 \pm 4$ & $90 \pm 2$ & 1.1 \\
\hline
\end{tabular}

${ }^{\text {aAdded concentration }}$

- Below method LOQs 
Table 4. Summary of IMI concentrations, with standard deviations, in positive wine samples, $\mathrm{n}=3$ replicates.

\begin{tabular}{cc}
\hline Code & Concentration $\left(\mathrm{ng} \mathrm{mL}^{-1}\right) \pm \mathrm{SD}$ \\
\hline 1 & $17.0 \pm 0.5$ \\
2 & $2.7 \pm 0.1$ \\
3 & $14.6 \pm 0.3$ \\
4 & $7.5 \pm 0.2$ \\
5 & $4.8 \pm 0.1$ \\
6 & $1.3 \pm 0.1$ \\
7 & $14.6 \pm 0.4$ \\
8 & $8.3 \pm 0.3$ \\
9 & $2.8 \pm 0.1$ \\
10 & $29.1 \pm 0.4$ \\
11 & $28.0 \pm 1.1$ \\
12 & $33.8 \pm 2.0$ \\
Mean & 13.7 \\
Median & 11.5 \\
\hline
\end{tabular}


Supplementary information to manuscript:

Evaluation of supercritical fluid chromatography accurate mass spectrometry for neonicotinoid compounds determination in wine samples

L. Pérez-Mayán, M. Cobo-Golpe, M. Ramil, R. Cela, I. Rodríguez

Department of Analytical Chemistry, Nutrition and Food Sciences. Institute for

Research and Food Analysis (IIAA). Universidade de Santiago de Compostela, 15782-

Santiago de Compostela, Spain.

*corresponding author: M. Ramil

e-mail:maria.ramil@usc.es

Tel. 0034881814466 
Fig. S1. Chemical structures, CAS numbers and octanol-water partition coefficients of neonicotinoids.<smiles>CN/C(=N/[N+](=O)[O-])NCC1CCOC1</smiles>

Dinotefuran (DIN) CAS number: $165252-70-0$ Log Kow: $-0.381 \pm 0.446$<smiles>N#CN=C1SCCN1Cc1ccc(Cl)nc1</smiles>

Thiacloprid (THC) CAS number: $111988-49-9$ Log Kow: $2.201 \pm 0.463$<smiles>CCN(Cc1ccc(Cl)nc1)/C(=C/[N+](=O)[O-])NC</smiles>

Nitenpyram (NITE) CAS number: $150824-47-8$ Log Kow: 0.511 \pm 0.376<smiles>[2H]C([2H])([2H])NC(=N[N+](=O)[O-])NCc1cnc(Cl)s1</smiles>

Clothianidin- $\mathrm{d}_{3}$ (CLO-d $\mathrm{d}_{3}$ ) CAS number: $1262776-24-8$<smiles>CC(=NC#N)N(C)Cc1ccc(Cl)nc1</smiles>

Acetamiprid (ACE) CAS number: 135410-20-7 Log Kow: $1.554 \pm 0.445$<smiles>O=[N+]([O-])Nc1nccn1Cc1ccc(Cl)nc1</smiles>

Imidacloprid-olefin (IMI-OLE) CAS number: 115086-54-9 Log Kow: $1.075 \pm 0.444$<smiles>CN1COCN(Cc2cnc(Cl)s2)C1=N[N+](=O)[O-]</smiles>

Thiamethoxam (THM) CAS number: 153719-23-4 Log Kow: $0.162 \pm 0.764$<smiles>[2H]C1([2H])N=C(N[N+](=O)[O-])N(Cc2ccc(Cl)nc2)C1([2H])[2H]</smiles>

Imidacloprid-d 4 (IMI-d 4 ) CAS number: 1015855-75-0<smiles>CN=C(NCc1cnc(Cl)s1)N[N+](=O)[O-]</smiles>

Clothianidin (CLO) CAS number: 210880-92-5 Log Kow: $0.732 \pm 0.588$<smiles>O=[N+]([O-])NC1=NCCN1Cc1ccc(Cl)nc1</smiles>

Imidacloprid (IMI) CAS number: 138261-41-3 Log Kow: $0.330 \pm 0.463$<smiles>[2H]C1([2H])OC([2H])([2H])N(Cc2cnc(Cl)s2)C(=N[N+](=O)[O-])N1C</smiles>

Thiamethoxam- $\mathrm{d}_{4}\left(\right.$ THM- $\left.\mathrm{d}_{4}\right)$ CAS number:1331642-98-8 
Fig. S2A. Reversed-phase UPLC chromatogram of neonicotinoids.

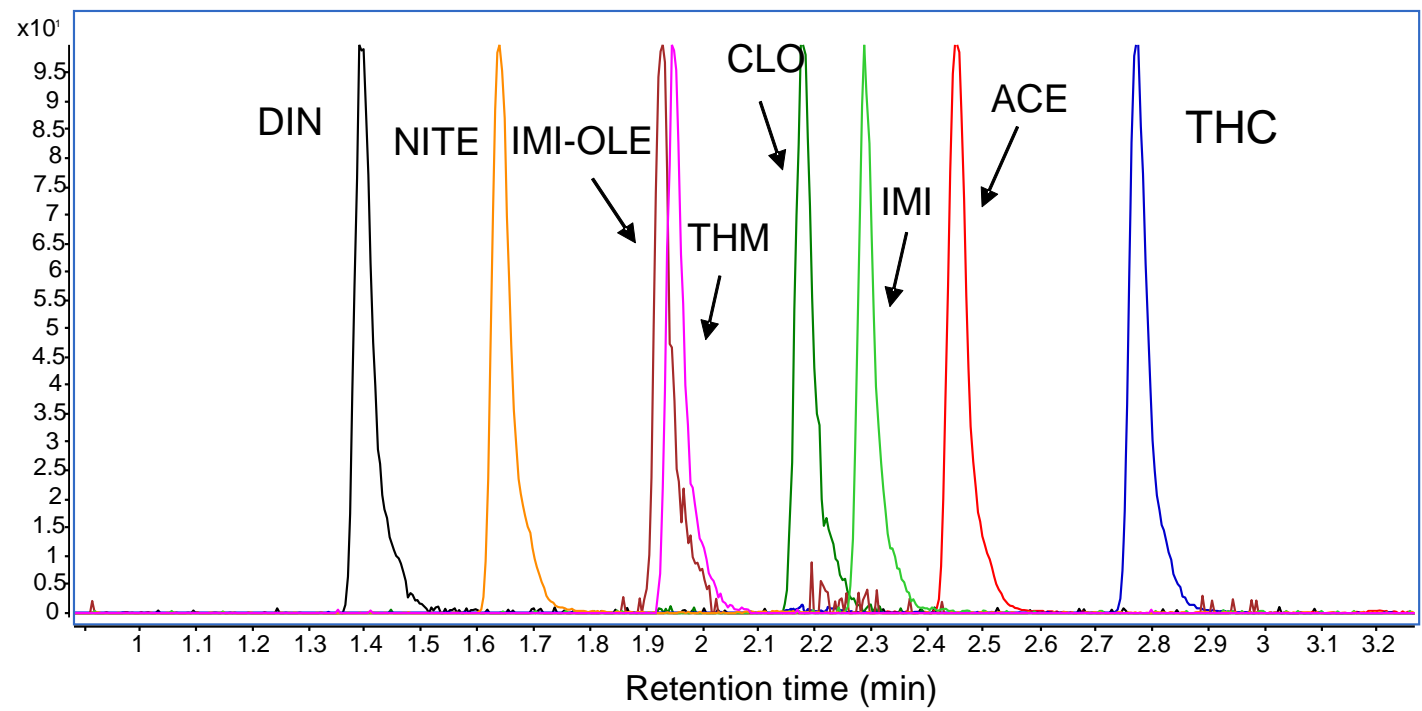

Fig. S2B. Plot of retention times in SFC (BEH silica column) and reversed-phase UPLC (X, axis).

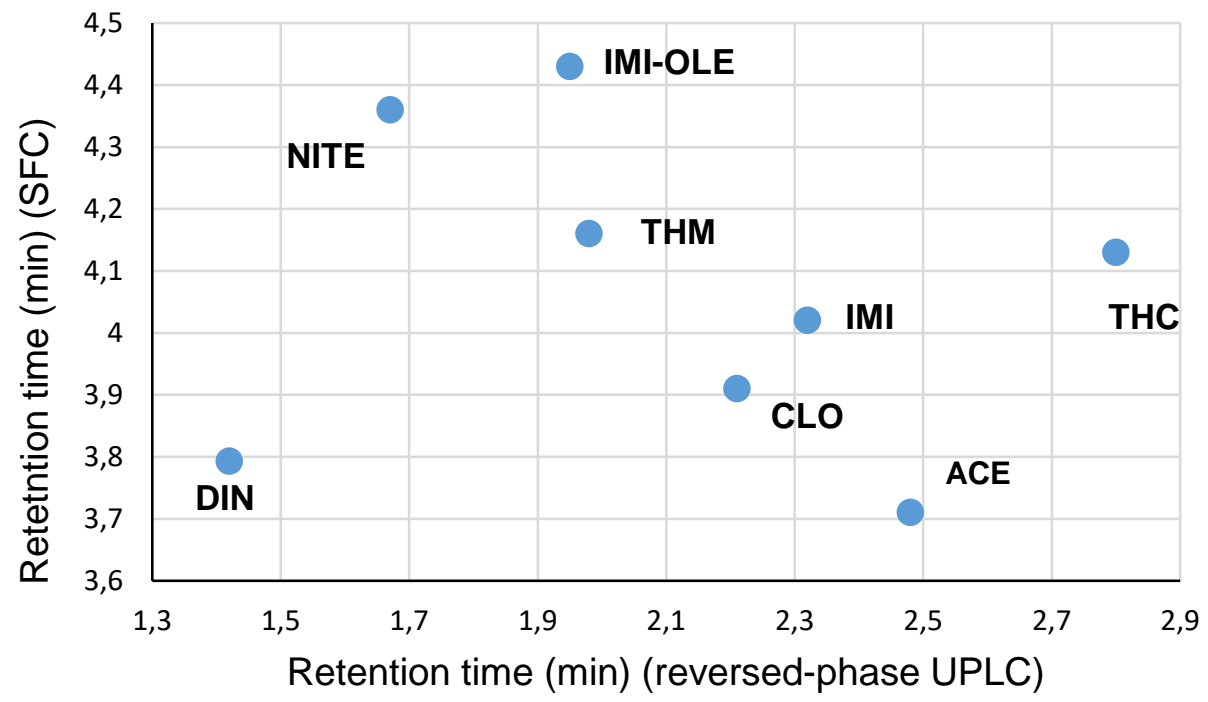


Fig. S3. Normalized responses for different pressures in the BPR module, $n=2$ replicates.

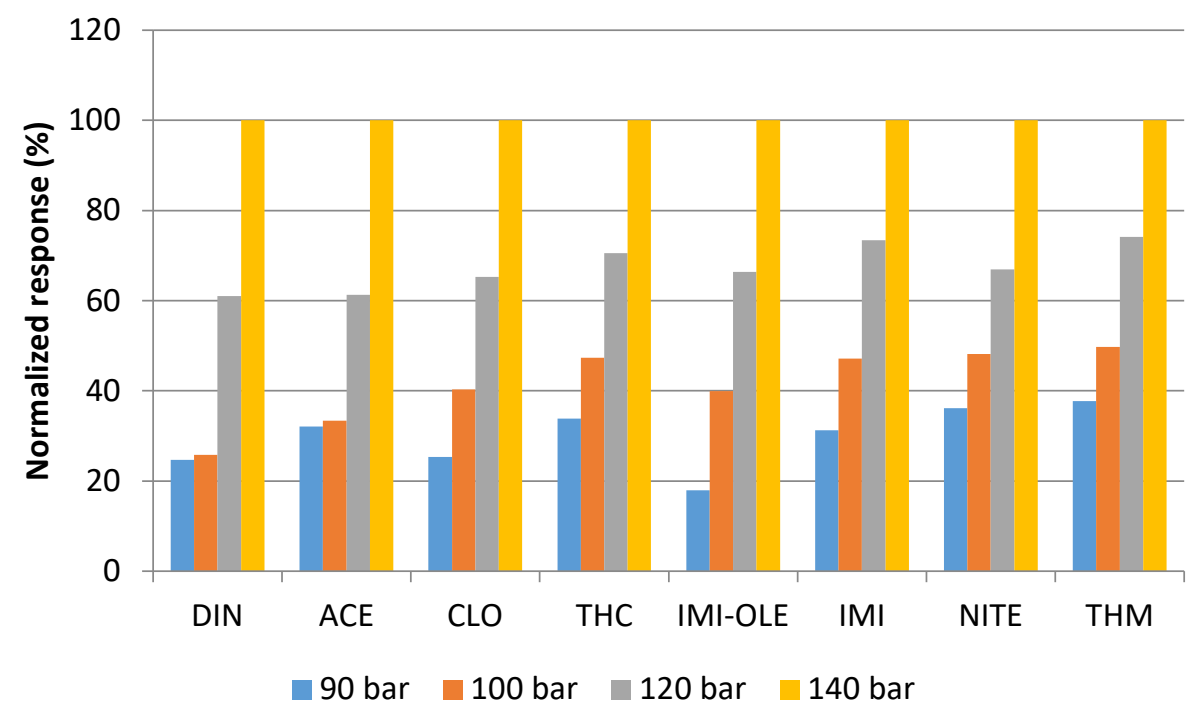

Fig. S4. Normalized responses as function of make-up $\left(\mathrm{MeOH}: \mathrm{H}_{2} \mathrm{O}, 25: 75\right)$ flow rate $\left(\mathrm{mL} \mathrm{min}^{-1}\right), \mathrm{n}=3$ replicates

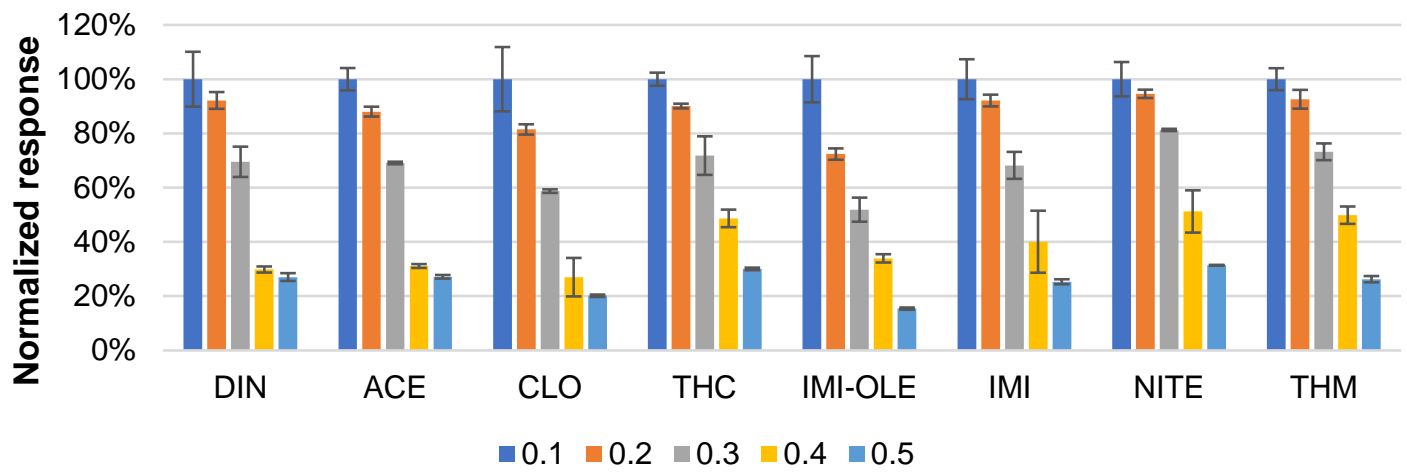


Figure S5. EIC chromatograms (SFC-TOF-MS) of the studied compounds at their LOQs (1 $\mathrm{ng} \mathrm{mL}^{-1}$, CLO, THC, IMI, NITE and THM; $10 \mathrm{ng} \mathrm{mL}^{-1}$, DIN, ACE, IMI-OLE).
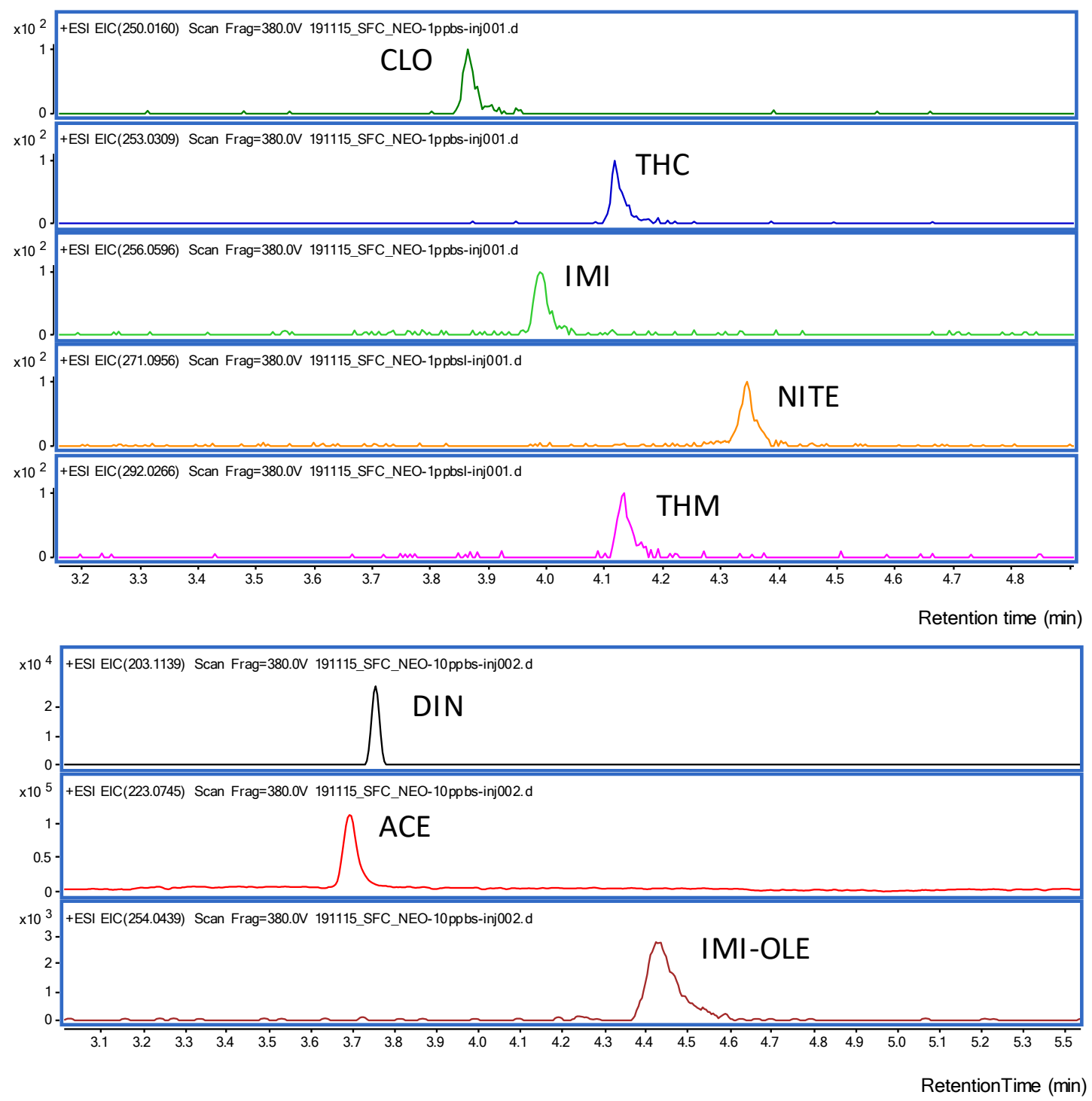
Fig. S6. Comparison of responses for consecutive injections ( $n=5$ replicates) of the same standard using SFC-ESI-MS and reversed-phase UPLC-ESI-MS as determination techniques. Injection volume $3 \mu \mathrm{L}$.

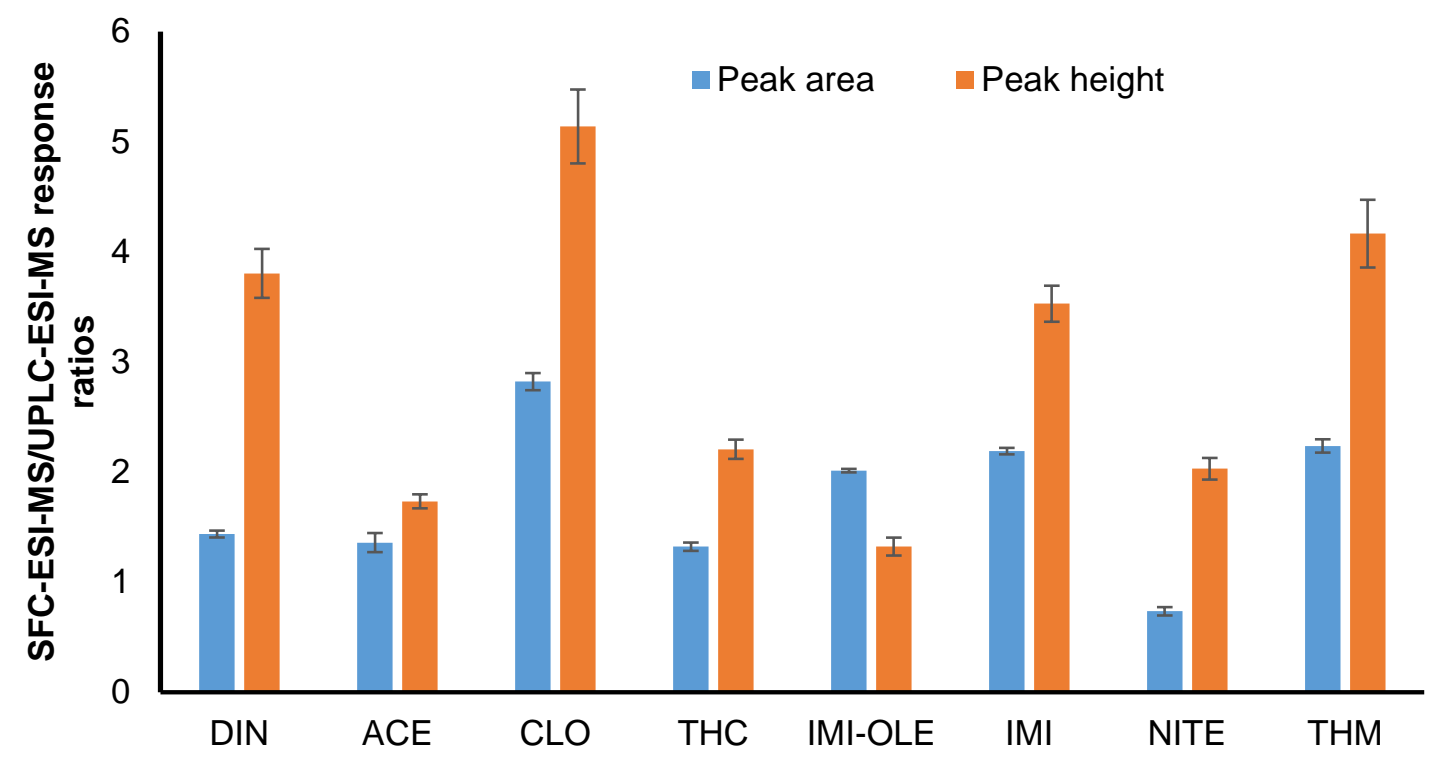

